\title{
Neutral Beyond-Standard-Model Higgs Searches in CMS
}

\section{Stefano Marcellini* On behalf of the CMS Collaboration}

I.N.F.N. Sezione di Bologna, Italy

E-mail: marcelliniabo.infn.it

\begin{abstract}
Searches for beyond the Standard Model neutral Higgs bosons at the CMS experiment are presented. The analyses are based on proton-proton collision data recorded by the CMS experiment at 7, 8 and $13 \mathrm{TeV}$ centre-of-mass energies. The exclusion limits determined by the null results of the searches are interpreted in the framework of models that include extensions of the standard Higgs sector.
\end{abstract}

Prospects for Charged Higgs Discovery at Colliders 3-6 October 2016

Uppsala, Sweden

\footnotetext{
* Speaker.
} 


\section{Introduction}

The physics program of the LHC run-1 data-taking period during 2011 and 2012 has been most strikingly affected by the discovery of a new particle by both the ATLAS and CMS collaborations, that has been identified as the Standard Model (SM) Higgs boson $H^{0}$. The measured properties of this particle are summarized in Ref. [1]. The two experiments measure consistent values of the mass of the particle, corresponding to a combined value $m_{H^{0}}=125.09 \pm 0.21$ (stat) \pm 0.11 (sys) $\mathrm{GeV}$. The evidence in the $\tau^{+} \tau^{-}$final state, together with the non-observation in the $\mu^{+} \mu^{-}$decay channel demonstrates a non-universal coupling of the new particle to fermions. Finally a detailed analysis of the spin, $\mathrm{CP}$ and decay width of the new particle, as well as a comprehensive analysis of its coupling structure have revealed consistency with the expectations for the SM Higgs boson within $\approx 10 \%$ precision.

Theoretical models beyond the SM predict the existence of additional Higgs bosons, in which the already discovered particle $H^{0}$ is one of several scalar bosons, either neutral or charged, in a mass range accessible by the LHC experiments. In this note the results of searches for additional heavy neutral Higgs bosons performed with data recorded by the CMS detector [2] are summarized. The analyzed data correspond to the full LHC run-1 dataset collected during 2011 and 2012 at $\sqrt{s}=7-8 \mathrm{TeV}$, and to the run-2 data at $\sqrt{s}=13 \mathrm{TeV}$ collected up to july 2016 . The run-1 data correspond to integrated luminosities of $5.1 \mathrm{fb}^{-1}$ (2011) and $19.7 \mathrm{fb}^{-1}$ (2012). The run-2 data correspond to $2.3-2.7 \mathrm{fb}^{-1}$ (2015) and $12.9 \mathrm{fb}^{-1}$ (2016). The searches are generally performed in a model independent approach, and exclusion limits in terms of production cross section times the corresponding decay branching ratio are presented. The results are interpreted according to beyond-SM (BSM) Higgs scenarios, which include the Two Higgs Doublet Model (2HDM), the Minimal Supersymmetric SM (MSSM) and the Singlet Model. The entire set of exclusion limits is used to constraint the parameters of such models.

\section{BSM Higgs scenarios}

From the most general 2HDM, two scenarios called type-I and type-II can be derived. In $2 \mathrm{HDM}$ of type-I, the $S U(2)_{L}$ doublets couple to both up- and down-type fermions equally; in 2HDM of type-II, one doublet couples exclusively to up-type and the other exclusively to downtype fermions. The model predicts the existence of five Higgs physical states: the three neutral $h$, $H$, and $A$, and the charged $H^{ \pm}$. The free parameters can be reduced to seven, and they are chosen to be $m_{h}, m_{A}, m_{H}, m_{H^{ \pm}}$, the mixing angle of the CP-even Higgs states $\alpha$, the ratio of the vacuum expectation values of the two doublets, $\tan \beta$, and the soft $Z_{2}-$ breaking mass parameter $m_{12}$. The two angles $\alpha$ and $\beta$ are substituted by $\cos (\beta-\alpha)$ and $\tan \beta$ without loss of generality. More details on the choice of the parameters can be found in [3]

The MSSM is a specific example of a $2 \mathrm{HDM}$ of type-II, in the framework of Supersymmetry. At tree level, the two free parameters $m_{A}$ and $\tan \beta$ fully constrain the Higgs sector. The Higgs sector of the MSSM has been studied extensively already before the advent of the LHC [4]. For the results presented in this note the $m_{h}^{\bmod +}$ and the hMSSM scenario [5][6] have been chosen. The $m_{h}^{\bmod +}$ scenario is a modification of the former $m_{h}^{\max }$ scenario, developed after the discovery of the $H^{0}$, to make $m_{h}$ compatible with $m_{H^{0}}$ within a theoretical uncertainty of $\pm 3 \mathrm{GeV}$ [7] in 
as much of the parameter space as possible. The hMSSM scenario has recently been introduced as an effective MSSM model, trading the precise knowledge of $m_{H^{0}}$. In this way $m_{h}=m_{H^{0}}$ is a manifest requirement of the scenario. This scenario is strictly valid for $m_{A}>130 \mathrm{GeV}$ and $\tan \beta<10$. A detailed discussion of the hMSSM scenario is given in Ref. [8].

\section{Indirect constraints from the coupling of $H^{0}$}

When interpreted as the $h$ boson, the predicted production and decay rates of the $H^{0}$ are particularly sensitive to the angles $\alpha$ and $\beta$. In the 2HDM these angles are free parameters. In the MSSM and hMSSM they can be expressed in terms of the free parameters $m_{A}$ and $\tan \beta$. Thus in both cases the allowed parameter space is constrained by the production and decay of the $H^{0}$.

Such constraints have been obtained using the CMS inputs to the combined ATLAS and CMS coupling analysis as presented in Ref. [1]. The existing data have been re-interpreted, without introducing modifications in the selected analyses. The production and decay rates of $h$ relative to the SM expectation have been varied using the formalism of leading-order coupling modifiers, $\kappa_{i}$, as defined in Ref. [9].

The $95 \%$ confidence level (CL) exclusion contours in the 2HDM of type-I and II, in the $\cos (\beta-\alpha)$-tan $\beta$ plane, are shown in Figure 1 as obtained from the observed couplings of the discovered $H^{0}$ boson. The observed exclusion contours are shown in black. The expected exclusion contours, assuming only the SM Higgs sector, are indicated by the red continuous line. Also shown are the expectations by the SM (indicated by the red dashed line) and the likelihood estimate (indicated by the black cross). The results reflect the high consistency of the $H^{0}$ properties with the expectation for the SM Higgs boson, and set severe constraints to the parameters of the model. The details of this analysis are described in [3].
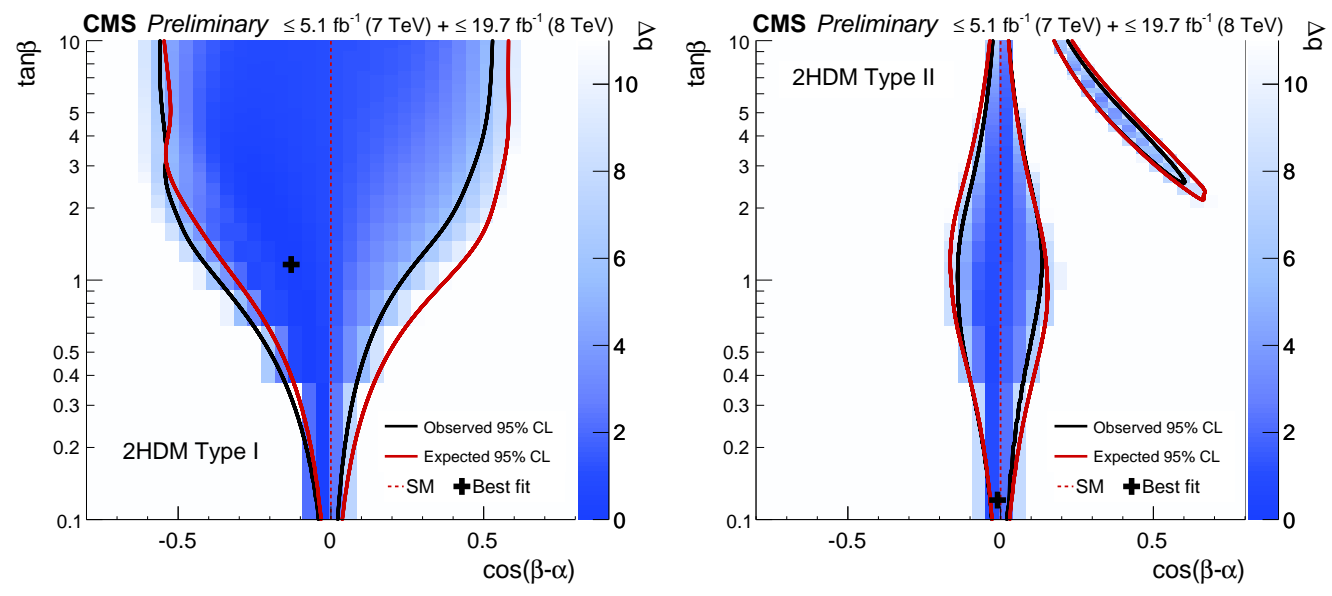

Figure 1: General constraints on the $2 \mathrm{HDM}$ parameter space obtained from the compatibility with the observed couplings of the $H^{0}$ when interpreted as the $h$ boson. The lines show the contours which restrict the allowed parameter space at the $95 \% \mathrm{CL}$ for a $2 \mathrm{HDM}$ of (left) type-I and (right) type-II. The observed constraints are shown in black. The expected constraints assuming just the SM Higgs sector are indicated by the red continuous line. 


\section{Direct searches}

A selected set of CMS results on the search of BSM neutral Higgs bosons obtained using run-1 data have been used to determine 95\% CL exclusion limits within the framework of 2HDM and MSSM models, as described in Ref. [3]. Such limits are shown in the $\tan \beta-m_{H}$ (2HDM) and $\tan \beta-m_{A}$ (MSSM) parameter space. Figure 2 (left) shows the 95\% CL exclusion contours in the 2HDM scenario (left) of type-I, as obtained by selected CMS analyses that have been performed on the LHC run-1 dataset. The colored filled areas correspond to the excluded regions. As previously discussed, by definition the whole parameter space that is displayed is mostly compatible with the constraints imposed by the coupling of $H^{0}$.

The 95\% CL exclusion contours of five direct searches in the MSSM $m_{h}^{\bmod +}$ scenario are displayed in Figure 2 (centre). Values up to $\tan \beta \approx 60$ are shown for masses up to $m_{A}=1 \mathrm{TeV}$. For larger values of $\tan \beta$ the predictions become generally unstable. The impact of the direct searches on the hMSSM model are shown in Figure 2 (right). The regions where the hMSSM is not strictly applicable are marked in gray. In the figure on the right, the constraint that is obtained from the compatibility of the scenario with the couplings of the $H^{0}$ is also shown. Further details on the interpretation of these exclusion limits can be found in Ref. [3].
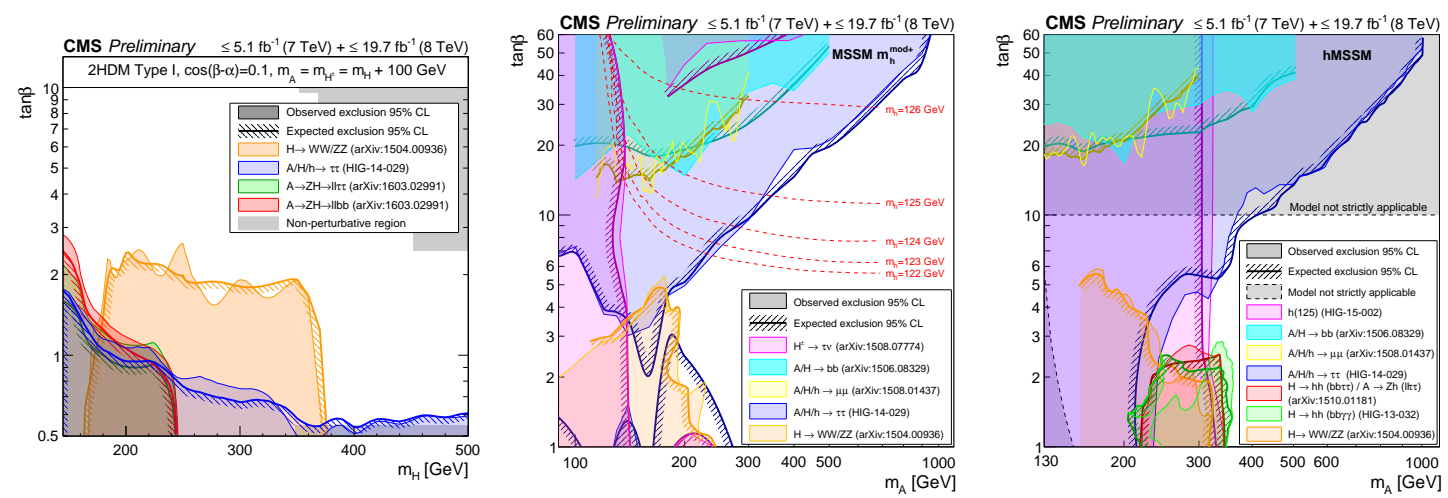

Figure 2: The 95\% CL exclusion contours in 2HDM scenarios of type-I (left), in the MSSM $m_{h}^{\text {mod }+}$ scenario (centre), and in the hMSSM model (right), as obtained by selected CMS analyses that have been performed on the LHC run-1 dataset.

The search for neutral Higgs bosons in the two-fermion final state $p p \rightarrow h / H / A \rightarrow f^{+} f^{-}$ allows the test of the coupling of the extra bosons with down-type fermions. This is particularly interesting for 2HDM type-II and MSSM, which predict an enhancement of the coupling at large $\tan \beta$. For practical experimental reason the accessible final states are $\tau^{+} \tau^{-}, \mu^{+} \mu^{-}$and $b \bar{b}$. The production mechanism can occur either via gluon-gluon fusion and b-associated production. In this latter case the analyses require the presence of b-tagged jets in the event, not associated to the Higgs candidate decay.

The $\tau^{+} \tau^{-}$final state represents the golden channel for this type of search, being the best compromise between a relatively large decay branching ratio and a small background. These features make this analysis the most sensitive search to all three neutral Higgs bosons in the MSSM. The search is performed in the $\mu \mu, e \mu, \mu \tau_{h}, e \tau_{h}$ and $\tau_{h} \tau_{h}$ final state decays of the $\tau$ into electrons, muons, and hadrons. The presence of neutrinos in the final state introduces complications in the in- 
variant mass reconstruction, the resolution of which ranges between 10 and $20 \%$ of the di-tau mass, depending on the final state. To enhance the sensitivity of the analysis to the MSSM predictions, events are categorized according to the presence/absence of b-tagged jets. The coarse mass resolution justifies the use of single mass templates obtained from the simulation with PYTHIA [10], produced for several values of $m_{A}$. The analysis has been performed using data taken at $\sqrt{s}=7-8$ $\mathrm{TeV}$ [11], and it has been updated using data collected at $13 \mathrm{TeV}$ [12] for an integrated luminosity of $2.3 \mathrm{fb}^{-1}$. Model independent 95\% CL exclusion limits are obtained in terms of $\sigma \times B . R$. for the production and subsequent decay to a tau pair of a generic boson $\phi$ as a function of $m_{A}$, as shown in Figure 3 for (left) the gluon-fusion production, and (centre) the b-associated production mechanism. No excess is found up to a test mass of $3 \mathrm{TeV}$. The results can also be interpreted in the contest of the $m_{h}^{\bmod +}$ scenario of the MSSM, to set limits in the $m_{A}$-tan $\beta$ plane as shown in Figure 3 (right).
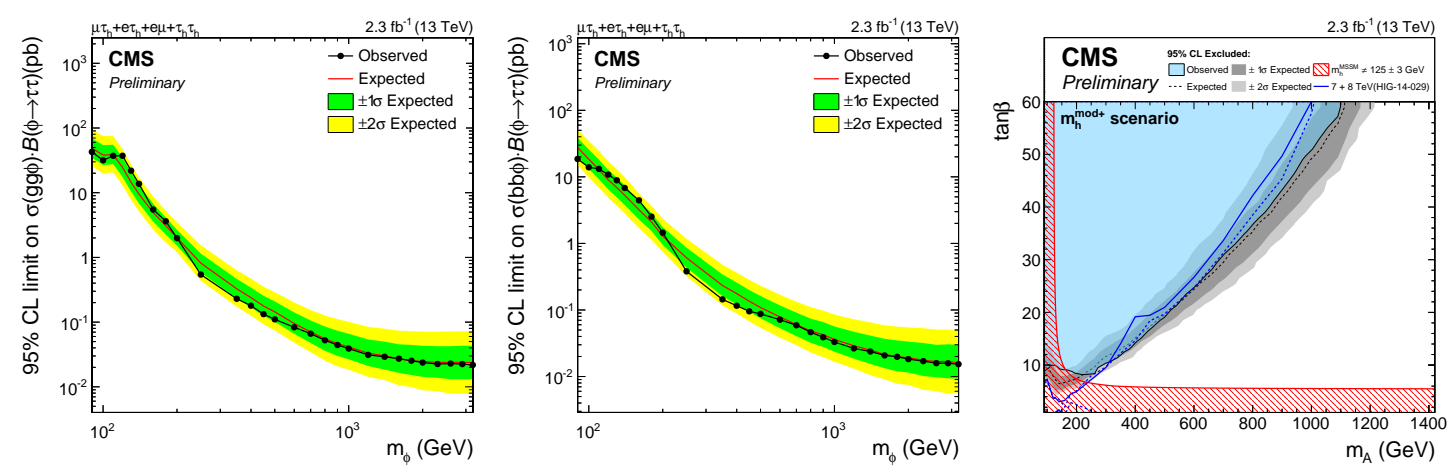

Figure 3: Expected and observed 95\% CL exclusion limits on cross-section times branching ratio for (left) the gluon fusion process and (centre) the b-associated production process of $p p \rightarrow \phi \rightarrow \tau \tau$. The model dependent exclusion limits in the $m_{A}$-tan $\beta$ for the $m_{h}^{\text {mod }}+$ scenario of the MSSM is shown in (right). The blue lines indicate the expected (dashed) and observed (solid) exclusions obtained from the most recent run1 CMS search for this channel [11], and the red contour indicates the region which does not yield a Higgs boson consistent with a mass of $125 \mathrm{GeV}$ within the theory uncertainties of $\pm 3 \mathrm{GeV}$.

The search for the $b \bar{b}$ final state benefits from the high decay branching ratio, but it suffers from the very high b-jet background at LHC. The analysis, performed using data collected at 8 $\mathrm{TeV}$ [13], searches for heavy scalar bosons in b-associated production. For this reason at least $3 \mathrm{~b}-$ tagged jets are required in the event. The mass of the Higgs candidate is reconstructed from the two leading b-jets in the event, with a resolution of about 20\%. Since in the MSSM the $A$ and $H$ bosons are degenerate in mass, a potential signal can be interpreted as the incoherent sum of these two individual contributions. The search is performed in the mass range from 100 to $900 \mathrm{GeV}$. Since no excess is observed, the 95\% CL model independent exclusion limit can be translated into an exclusion limit in the $m_{A}$-tan $\beta$ plane within the MSSM model. A different analysis [14] searching for the same final state has been performed by the CMS experiment using data collected at $13 \mathrm{TeV}$. A generic neutral scalar decaying to bottom-quark pairs is excluded in the mass range from 500 to $1200 \mathrm{GeV}$.

The excellent muon invariant mass resolution of the CMS experiment has been exploited to search for BSM neutral scalars decaying to $\mu^{+} \mu^{-}$pairs at 7-8 TeV centre-of-mass energies [15]. Both the gluon fusion and the b-associated production mechanisms are considered. Despite the 
very low decay branching ratio, the analysis sensitivity is compatible with the $b \bar{b}$ final state in the mass range where the available statistics is large enough to perform a meaningful measurement. Model independent limits are determined for the $\sigma \times B . R$. of a generic neutral scalar decaying to a muon pair for masses up to $500 \mathrm{GeV}$. The results are also interpreted in the $m_{h}^{\text {mod }}{ }^{+}$scenario of the MSSM model as exclusion limits in the $m_{A}$-tan $\beta$ plane, in the mass range $m_{A}<300 \mathrm{GeV}$.

Searches for the decay of a heavy neutral scalar $X$ decaying to a pair of light $H^{0}$ bosons have been performed. In the $\mathrm{SM}$, at $\sqrt{s}=13 \mathrm{TeV}$ the production cross section of a $H^{0}$ pair is about $30 \mathrm{fb}$. However the decay branching ratio of the heavy $X$ particle in two SM Higgs bosons can become significantly large in the singlet model, translating into a resonance with mass $m_{X}$. The non resonant production mechanism, which is not addressed in this note, can also show indications of new physics via virtual loops or modified couplings, and it will acquire importance in the future, with larger integrated luminosity.

The search for a generic neutral resonance $X$ of either spin 0 or 2, decaying to a SM Higgs boson pair, where one $H^{0}$ decays to a $b \bar{b}$ pair and the other to a $\gamma \gamma$ pair has been performed by the CMS experiment at $\sqrt{s}=13 \mathrm{TeV}$ and it is described in Ref. [16]. A benchmark used for this analysis is the Randall-Sundrum (RS) model of warped extra dimensions (WED) [17], that predicts new spin-0 (radions) or spin-2 (excited gravitons) particles that could decay to a SM Higgs pair if their mass is heavier than $2 m_{H^{0}}$. The analysis is restricted to the mass range $250<m_{X}<900$ $\mathrm{GeV}$, as for higher mass values the Higgs bosons become very boosted, thus reducing its detection efficiency. The exclusion limits are shown in Figure 4. The expectations for a spin-0 radion (left) and spin-2 graviton (excited graviton) based on the RS predictions are superimposed.
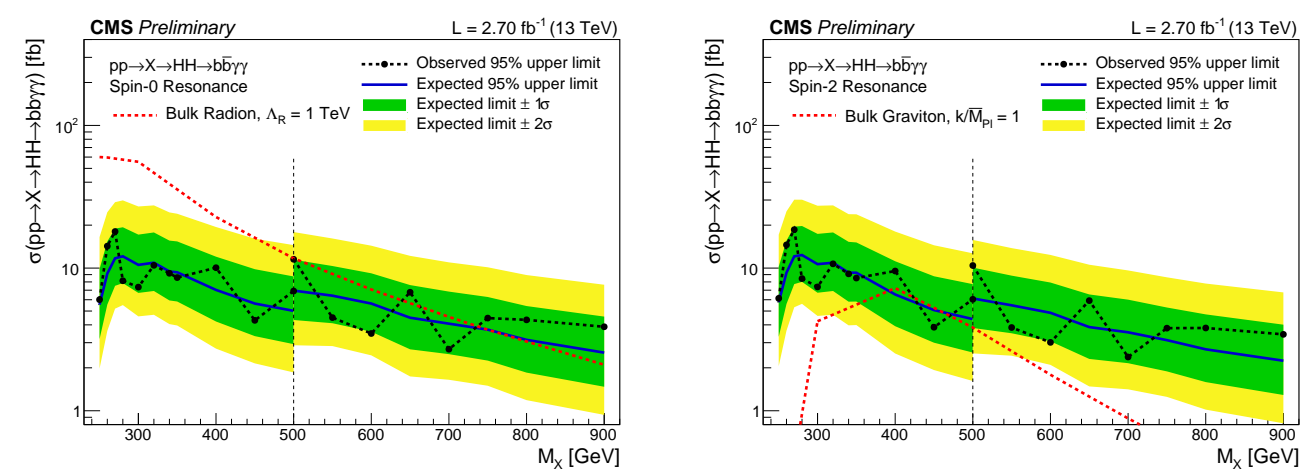

Figure 4: Expected and observed 95\% CL upper limits on the production of $p p \rightarrow X \rightarrow H^{0} H^{0} \rightarrow b \bar{b} \gamma \gamma$ for the resonant analysis, assuming (left) spin- 0 and (right) spin-2 for the resonance. Cross sections predicted by the RS model are shown (dashed red lines). The SM branching ratio for $H^{0} H^{0} \rightarrow b \bar{b} \gamma \gamma$ is assumed.

The search for the process $p p \rightarrow X \rightarrow H^{0}(b \bar{b}) H^{0}(b \bar{b})$ performed by the CMS experiment at $\sqrt{s}=13 \mathrm{TeV}$ is described in Ref. [18]. The analysis is performed over the mass range $260<$ $m_{X}<1200 \mathrm{GeV}$. However, since the momenta and angles of the decay products of such a resonance change substantially over this range and in order to maximize the sensitivity of this search, different event selection criteria are used for the two main kinematic regions: the low-mass region (LMR) for resonance mass hypotheses from $260 \mathrm{GeV}$ to $400 \mathrm{GeV}$, and the medium-mass region (MMR) for masses from $400 \mathrm{GeV}$ to $1200 \mathrm{GeV}$. The $400 \mathrm{GeV}$ transition threshold between the LMR and the MMR has been chosen to maximize the expected sensitivity. The QCD multi-jet background 
is modeled in data by studying parametric fits in sideband regions. At least four b-tagged jets are required in the event, and the b-jets are suitably paired according to dedicated kinematic constraints to reconstruct the mass of the two $H^{0}$ bosons, $m_{H 1}$ and $m_{H 2}$. Signal and sidebands regions are defined according to the quantity

$$
\chi^{2}=\left(\frac{m_{H 1}-115 \mathrm{GeV}}{\sigma_{H}}\right)+\left(\frac{m_{H 2}-115 \mathrm{GeV}}{\sigma_{H}}\right)
$$

which is shown in Figure5 (left) as a function of the two Higgs boson masses. The 95\% CL exclusion limits in terms of $\sigma \times B . R$. are shown in Figure 5 (right) as a function of the $m_{X}$ mass hypothesis. The expectations for a Kaluza Klein (KK) graviton are superimposed.
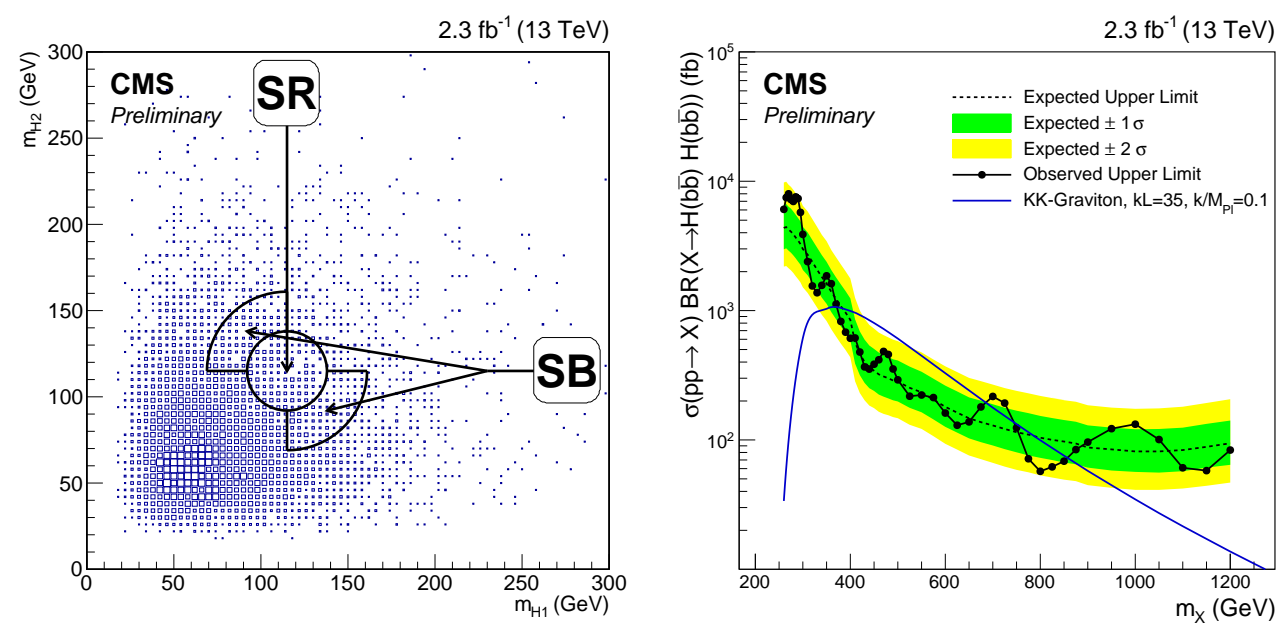

Figure 5: Illustration (left) of signal (SR) and side-band (SB) regions in the $m_{H 1}, m_{H 2}$ plane. The quantities $m_{H 1}$ and $m_{H 2}$ are the two reconstructed Higgs boson masses after b-tagging and kinematic selections for data. The observed and expected upper limits (right) on the cross section for a spin-2 resonance $X \rightarrow H(b \bar{b}) H(b \bar{b})$ at a $95 \% \mathrm{CL}$. Theoretical cross sections for the RS KK-Graviton, with $k / M_{P l}=0.1, k L=35$, decaying to four b-jets via Higgs bosons are overlaid.

\section{Di-boson final state: $X \rightarrow W W / Z Z$}

The search for a generic neutral $X$ resonance of unknown mass decaying to a di-boson pair $W^{+} W^{-}$or $Z Z$ allows the study of the low/intermediate $\tan \beta$ region in the mass range from $2 m_{W}$ to $2 m_{t}$, when interpreted in the $2 \mathrm{HDM}$ and MSSM models. Above $2 m_{t}$ the decay channel $X \rightarrow t \bar{t}$ would open up, thus significantly reducing the branching ratio to boson pairs. This channel is a good probe for singlet models, that predict the existence of an additional heavy neutral Higgs boson.

The CMS experiment has performed the search for the process $p p \rightarrow X \rightarrow Z(l l) Z(l l)$ using a fraction of the data collected at $\sqrt{s}=13 \mathrm{TeV}$ in 2016, as described in Ref. [19]. The analysis is the extension of the four-lepton SM Higgs search at higher mass values. The invariant mass distribution of the four lepton final state (eеeе, еe $\mu \mu, \mu \mu \mu \mu)$ is reported in Figure 6 (left). In addition to the peak corresponding to the SM Higgs boson with $m_{H 0}=125 \mathrm{GeV}$, the distribution 
is populated by events with masses higher than $2 m_{Z}$, where the search for heavy additional Higgs bosons is performed. The production mechanism of the additional Higgs boson can be either gluon fusion (gg) or vector-boson fusion (VBF), which also includes the process $V \rightarrow V H$. The fraction of gg produced events, $f_{g g}$ is then related to the fraction of VBF-VH events, $f_{V B F}$ by the relation: $f_{g g}=1-f_{V B F}$. The search is performed for various Higgs widths assumptions. Figure 6 (right) shows the expected and observed 95\% CL exclusion limits of $\sigma \times B . R$. as a function of the Higgs mass hypothesis $m_{X}$, when the fraction $f_{V B F}$ is left to vary in the fit. No significant excess is observed in the mass range $130-2530 \mathrm{GeV}$.
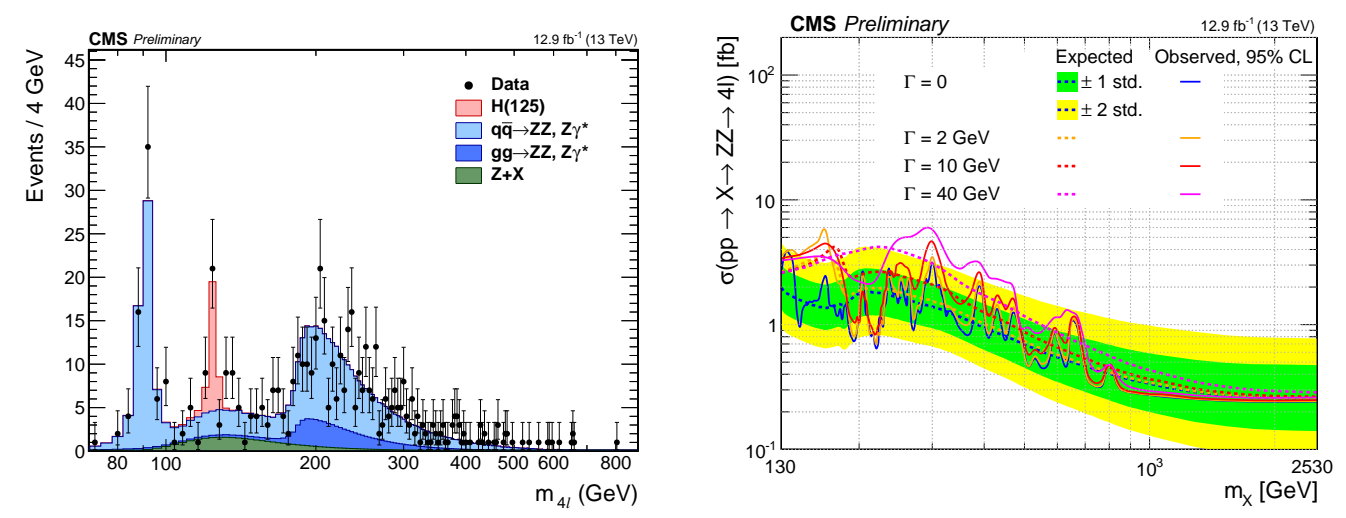

Figure 6: (left) Distribution of the four-lepton reconstructed invariant mass $m_{4 l}$ in the full mass range, including the SM-Higgs peak. (right) Observed and expected upper limit at the 95\% CL on the $X \rightarrow Z Z \rightarrow 4 l$ cross section times the decay branching ratio as a function of $m_{X}$ at several $\Gamma_{X}$ values, with $f_{V B F}$ floated.

\section{Conclusions}

The discovery of the neutral resonance having a mass of about $125 \mathrm{GeV}$ was a major achievement in LHC physics. This resonance has been identified as the SM Higgs boson, as its properties and couplings with fermions and bosons are very consistent with the expectation for this particle. However, according to BSM scenarios, it is possible that the $125 \mathrm{GeV}$ particle is part of an extended Higgs sector which would encompass the existence of additional neutral and charged particles of unknown mass. The measurements performed by the CMS and ATLAS experiments on the SM Higgs properties can be used to severely constrain such new models, that have to be compatible with the existence of the observed $H^{0}$. Moreover, direct searches of new neutral scalar particles have been performed by the CMS collaboration using data of the LHC collected during run-1 and run-2, to assess exclusion limits on the production and decay of such hypothetical new particles. The most stringent exclusion limits currently come from the $\tau \tau$ decay final state, but precise measurements on $X \rightarrow H^{0} H^{0}$ and $X \rightarrow W W / Z Z$ processes also contribute to set limits on the production and decay of such particles.

\section{References}

[1] CMS Collaboration, "Measurements of the Higgs boson production and decay rates and constraints on its couplings from a combined ATLAS and CMS analysis of the LHC pp collision data at $\sqrt{s}=7$ and 8 TeV", CMS Physics Analysis Summary CMS-PAS-HIG-15-002, 2015. 
[2] CMS Collaboration, “The CMS experiment at the CERN LHC”, JINST 3 (1008) S08004, doi:10.1088/1748-0221/3/08/S08004.

[3] CMS Collaboration, "Summary results of high mass BSM Higgs searches using CMS run-I data", CMS Physics Analysis Summary, CMS-PAS HIG-16-007 (2016).

[4] P. Bechtle et al., "Probing the Standard Model with Higgs signal rates from the Tevatron, the LHC and a future ILC”, JHEP 11 (2014) 039, doi:10.1007/JHEP11(2014)039, arXiv:1403.1582[hep-ph].

[5] A. Djouadi et al., “The post-Higgs MSSM scenario: Habemus MSSM?”, Eur. Phys. J.C73 (2013) 2650, doi:10.1140/epjc/s10052-013-2650-0, arXiv:1307.5205[hep-ph].

[6] A. Djouadi et al., "Fully covering the MSSM Higgs sector at the LHC”, JHEP 06 (2015) 168, doi:10.1007/JHEP06(2015)168, arXiv:1502.05653[hep-ph].

[7] G. Degrassi et al., "Towards high precision predictions for the MSSM Higgs sector”, Eur.Phys.J.C28 (2003) 133143, doi:10.1140/epjc/s2003-01152-2, arXiv:0212020[hep-ph].

[8] LHCHXSWG Collaboration, "Benchmark scenarios for low $\tan \beta$ in the MSSM", public note LHCHXSWG-2015-002, 2015.

[9] LHC Higgs Cross Section Working Group et al., "Handbook of LHC Higgs Cross Sections: 3. Higgs Properties”, CERN-2013-004 (CERN, Geneva, 2013) arXiv:1307.1347[hep-ph].

[10] T. Sjöstrand, S. Mrenna, and P. Skands, "PYTHIA 6.4 physics and manual", JHEP 05 (2006) 026, arXiv:0603175[hep-ph].

[11] CMS Collaboration, "Search for neutral MSSM Higgs bosons decaying to a pair of tau leptons in pp collisions”, JHEP 10 (2014) 160, arXiv:1408.3316[hep-ex].

[12] CMS Collaboration, "Search for a neutral MSSM Higgs boson decaying into $\tau \tau$ at $13 \mathrm{TeV}$ ", CMS Physics Analysis Summary, CMS-HIG-16-006 (2016).

[13] CMS Collaboration, "Search for Neutral MSSM Higgs Bosons Decaying into A Pair of Bottom Quarks”, JHEP 11 (2015) 071, doi:10.1007/JHEP11(2015)071, arXiv:1506.08329[hep-ex].

[14] CMS Collaboration, "Search for a narrow heavy decaying to bottom quark pairs in the $13 \mathrm{TeV}$ data sample”, CMS Physica Analysis Summary, CMS-HIG-16-025 (2016).

[15] CMS Collaboration, "Search for neutral MSSM Higgs bosons decaying to $\mu^{+} \mu^{-}$in pp collisions at $\sqrt{s}=7$ and 8 TeV", Phys. Lett. B752 (2016) 221246, doi:10.1016/j.physletb.2015.11.042, arXiv:1508.01437[hep-ex].

[16] CMS Collaboration, "Search for $H(b \bar{b}) H(\gamma \gamma)$ decays at $\sqrt{s}=13 \mathrm{TeV}$ ", CMS Physics Analysis Summary, CMS HIG-16-032 (2016).

[17] H. Davoudiasl, J. L. Hewett, and T. G. Rizzo, "Phenomenology of the Randall-Sundrum Gauge Hierarchy Model”, Phys. Rev. Lett. 84 (2000), no. SLAC-PUB-8241, 2080, doi:10.1103/PhysRevLett.84.2080, arXiv:9909255[hep-ph].

[18] CMS Collaboration, "Search for resonant pair production of Higgs bosons decaying to two bottom quark-antiquark pairs in proton-proton collisions at 13 TeV”, CMS Physics Analysis Summary, CMS HIG-16-002 (2016).

[19] CMS Collaboration, "Measurements of properties of the Higgs boson and search for an additional resonance in the four-lepton final state at $\sqrt{s}=13 \mathrm{TeV}$ ", CMS Physics Analysis Summary, CMS HIG-16-033 (2016). 